\title{
Theoretical and experimental investigation of the magnetic properties of polyvinylidene fluoride and magnetite nanoparticles-based nanocomposites
}

\author{
M. A. Ramazanov ${ }^{1}$ A. M. Maharramov ${ }^{1} \cdot$ R. A. Ali-zada ${ }^{2}$ H. A. Shirinova ${ }^{1} \cdot$ F. V. Hajiyeva ${ }^{1}$
}

Received: 9 March 2018/Accepted: 22 May 2018 / Published online: 28 May 2018

(c) The Author(s) 2018

\begin{abstract}
In the present study, the effect of size distribution of magnetite nanoparticles in a PVDF matrix on the magnetic properties of PVDF $+\mathrm{Fe}_{3} \mathrm{O}_{4}$ nanocomposites was experimentally and theoretically investigated. The size distribution of nanoparticles in polymer matrix and morphology of the nanocomposites were studied by the means of scanning electron microscopy and atomic force microscopy. It was found that when the $\mathrm{Fe}_{3} \mathrm{O}_{4}$ nanoparticles are introduced into the polymer matrix, their coagulation takes place. The increase in the size of the particles depends on their concentration in the polymer matrix, the type of polymer (polar, non-polar, its viscosity, etc.), reaction temperatures, etc. In addition, when $\mathrm{Fe}_{3} \mathrm{O}_{4}$ nanoparticles are introduced into the polymer network, the oxidation of the surface layer of particles occurs and the magnetic size decreases. Consequently, the reduced magnetic properties may also be observed. The hysteresis loops have been recorded in small magnetic field range. It was found that the magnetic hysteresis parameters depend on the size and concentration of $\mathrm{Fe}_{3} \mathrm{O}_{4}$ nanoparticles. Theoretical calculations were compared with experimental results obtained from $M(H)$ measurements. The reasons of differences between theoretical and experimental results have been explained.
\end{abstract}

Keywords Magnetite $\cdot$ Nanocomposite $\cdot$ Polymer $\cdot$ Hysteresis $\cdot$ Polyvinylidene fluoride

\section{Introduction}

Magnetic nanomaterials are drawing increased attention due to their remarkable physical properties. Superparamagnetic iron oxide nanoparticles, especially magnetite phase and nanocomposites based on $\mathrm{Fe}_{3} \mathrm{O}_{4}$ nanoparticles, have attracted interest from research due to possible applications of such materials in various fields $[1,2]$. The combination of different materials allows to make completely new composite materials with a wide range of functional properties: mechanical, chemical, electrical, magnetic, optical and many others [3]. Among these nanomaterials, thermoplastic polymer-based magnetic

M. A. Ramazanov

mamed_r50@mail.ru; nanomaterials@bsu.edu.az

1 Baku State University, Z. Khalilov Str. 23, AZ 1148 Baku, Azerbaijan

2 Institute of Physics, National Academy of Sciences of Azerbaijan, Pr. H. Javid 131, Baku, Azerbaijan nanocomposites attract significant academic and industrial interest because of their relatively easy and low-cost producing technology.

The formation of unique properties of polymer-based magnetic nanocomposites depends on many factors such as particles size and shape, degree of dispersion, concentration.

The determination of the size of the $\mathrm{Fe}_{3} \mathrm{O}_{4}$ nanoparticles before and after their introduction into the polymer matrix has been investigated in our early works [4]. The change in nanoparticle size depends on the type of polymer.

In the presented work, the dependence of the average size of $\mathrm{Fe}_{3} \mathrm{O}_{4}$ nanoparticles in the polymer matrix on its volume content and the dependence of the magnetic properties of the composites (saturation magnetization, residual magnetization, coercive force, etc.) on the size and concentration of the filler have been investigated. 


\section{Experimental details}

\section{Materials}

Polyvinylidene fluoride (PVDF) is a polar polymer that has a density of $1.78 \mathrm{~g} / \mathrm{cm}^{3}$ at $25^{\circ} \mathrm{C}$, melting point at the $T=177^{\circ} \mathrm{C}$.

Magnetite nanoparticles were obtained by co-precipitation method in an alkaline medium. The average nanoparticle size is $7-15 \mathrm{~nm}[1,2]$.

\section{Methods}

\section{Scanning electron microscopy (SEM)}

The distribution of magnetite nanoparticles in the polymer matrix has been studied by scanning electron microscopy (SEM, JEOL JSM-7600 F). Scanning was carried out in SEI mode with an accelerating voltage of $15 \mathrm{kV}$ and a working distance of $4.5 \mathrm{~mm}$.

\section{AFM analysis}

The morphology of the nanocomposites was studied using atomic force microscopy Integra Prima (NT-MDT, Zelenograd). For the scan used special silicon cantilevers fabricated by plasma etching method with the needle radius of curvature of $20 \mathrm{~nm}$ and the resonance frequency was $1-5 \mathrm{~Hz}$. Scan area was $2 \times 2 \mathrm{~mm}$. The measurements were taken in the semi-contact microscopy mode in air; the amplitude of cantilever's oscillation was fixed while determining the surface topography. The scanning speed and the number of scanned lines of the image are 256 and $1,969 \mathrm{~Hz}$, respectively.

\section{Method for studying magnetic properties}

Measurements: Magnetization curves acquired at $300 \mathrm{~K}$ (room temperature) by a Quantum Design SQUID magnetometer in the field range $\pm 50 \mathrm{kOe}$. The magnetization is reported per gram of measured sample.

\section{Synthesis of nanocomposites}

The polymer nanocomposite materials were prepared as follows: poly(vinylidene fluoride) was solved in dimethylformamide (DMF) solvent, at the room temperature. Magnetite nanoparticles were added into the polymer solution and stirred within $2 \mathrm{~h}$ in order to prepare a homogeneous mixture. In order to remove the solvent from the mixture, it was evaporated within 1 day.
The samples of nanocomposites were obtained by hot pressing method at melting point of PVDF under $15 \mathrm{MPa}$ pressure within $4 \mathrm{~min}$ on further cooling to room temperature [2].

\section{Results and discussion}

Figure 1 shows SEM images of nanocomposites based on $\mathrm{PVDF}+\mathrm{Fe}_{3} \mathrm{O}_{4}$ at various magnetite contents. As can be seen from the figure, for 1 and $3 \%$ volume contents of the filler, the sizes of magnetite nanoparticles in matrix are 13-21 nm and 29-40 nm, respectively. Using SEM images, approximately 100 particle sizes were taken for statistical parameters calculation.

The values of parameters in Gaussian and lognormal distribution of the $\mathrm{Fe}_{3} \mathrm{O}_{4}$ nanoparticles size in PVDF matrix are reported in Tables 1 and 2, respectively.

As can be seen from Table 2, the values of the standard deviation indicate a narrow lognormal particle size distribution. The values of the polydispersity index confirm the monodispersity $\mathrm{PVDF}+\mathrm{Fe}_{3} \mathrm{O}_{4}$ nanocomposites system. This shows that the size distribution of $\mathrm{Fe}_{3} \mathrm{O}_{4}$ particles in polymer matrix is fairly good and can be described by a lognormal distribution function:

$f(x)=\frac{1}{\sqrt{2 \pi} \sigma \cdot x} \cdot \exp \left(-\frac{(\ln x-\overline{\ln x})^{2}}{2 \sigma^{2}}\right)$

It is also clear from the table that the nature of the distribution of the $\mathrm{Fe}_{3} \mathrm{O}_{4}$ particles in the polymer matrix, as a function of their concentration and time, does not change. Only the distribution parameters: $\overline{\ln x}$ and $\sigma$ depend on the concentration of the particles in the polymer matrix and on the time. During coagulation, the dependence of the average particle diameter on time is analogous to the dependence of the average particle diameter on the number of particles (concentration), according to the theory [5].

Figure 2 shows the lognormal distribution of $\mathrm{Fe}_{3} \mathrm{O}_{4}$ nanoparticles in PVDF matrix at different volume contents.

The dependence of the average size of $\mathrm{Fe}_{3} \mathrm{O}_{4}$ nanoparticles in PVDF matrix on their concentrations was studied. Figure $3 \mathrm{a}, \mathrm{b}$ represents the dependence of the average particle sizes of $\mathrm{Fe}_{3} \mathrm{O}_{4}$ nanoparticles in the polymer matrix on volume content.

As can be seen from Fig. 3a, b, the dependence curve of the $\mathrm{Fe}_{3} \mathrm{O}_{4}$ particles diameter on the concentration in PVDF matrix is complicated. At low concentrations, this dependence for PVDF matrix is weak. This is due to the particle and polymer properties. Coagulation of particles also depends on the viscosity of the polymer which it has during the preparation of the composite. In a medium with a lower viscosity, the coagulation process will happen faster. As 

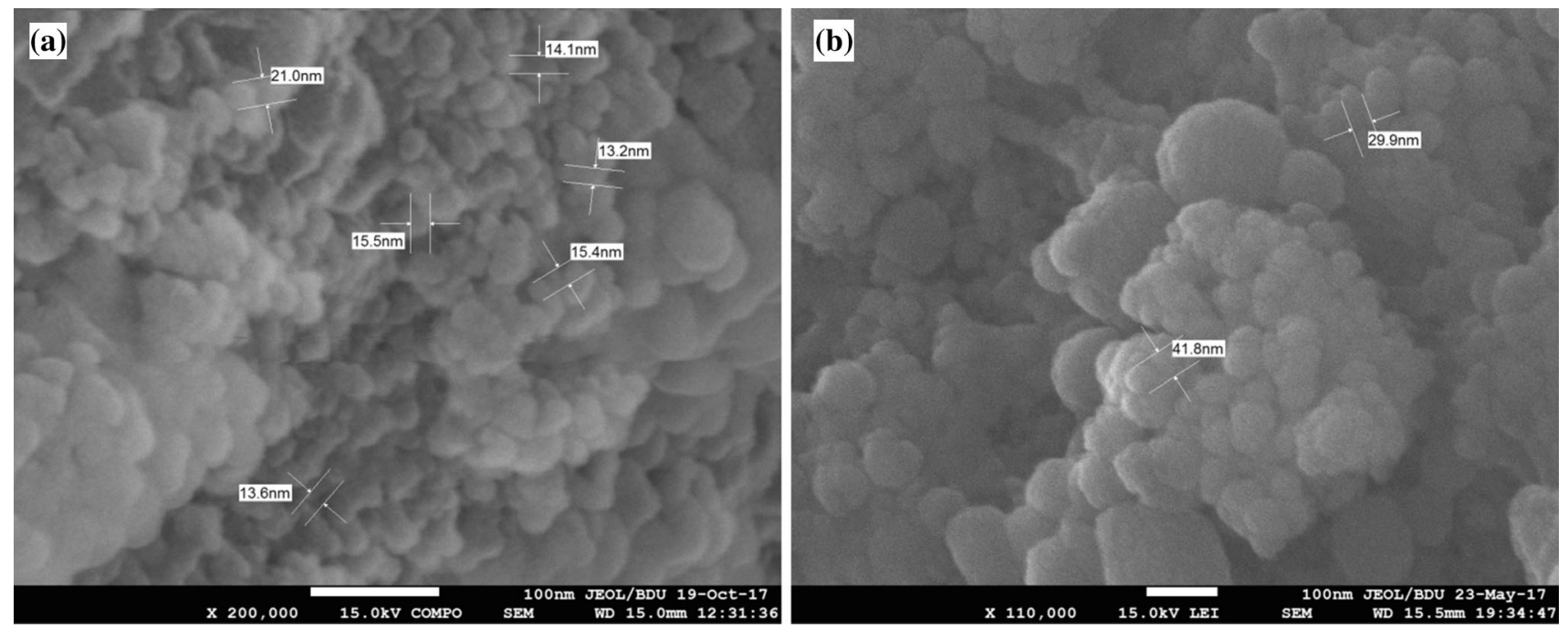

Fig. 1 SEM images of nanocomposites based on PVDF $+\mathrm{Fe}_{3} \mathrm{O}_{4}$. a PVDF $+1 \%$ vol $\mathrm{Fe}_{3} \mathrm{O}_{4}, \mathbf{b} \mathrm{PVDF}+3 \%$ vol $\mathrm{Fe}_{3} \mathrm{O}_{4}$

Table 1 Geometric values of the parameters (distribution interval, average value $(\bar{d})$, standard deviation $(\sigma)$, asymmetry (as), excess $(\varepsilon)$ and polydispersity index (PDI)) of $\mathrm{Fe}_{3} \mathrm{O}_{4}$ particles size distribution in PVDF matrix

\begin{tabular}{lccccr}
\hline Sample & $d_{\min }-d_{\max }(\mathrm{nm})$ & $\bar{d}(\mathrm{~nm})$ & $\sigma$ & GA & $\varepsilon$ \\
\hline pvdf $+1 \% \mathrm{Fe}_{3} \mathrm{O}_{4}$ & $13.2-57.2$ & 23.83 & 1.403 & 1.547 & 2.264 \\
pvdf $+3 \% \mathrm{Fe}_{3} \mathrm{O}_{4}$ & $17-54.2$ & 27.92 & 1.196 & 1.22 & 1.789 \\
\hline
\end{tabular}

Table 2 The logarithmic values of the parameters (distribution interval, average value $(\bar{d})$, standard deviation $(\sigma)$, asymmetry (as), excess $(\varepsilon)$ and polydispersity index (PDI)) of $\mathrm{Fe}_{3} \mathrm{O}_{4}$ particles size distribution

\begin{tabular}{|c|c|c|c|c|c|c|}
\hline sample & $d_{\min }-d_{\max }(\mathrm{nm})$ & $\bar{d}(\mathrm{~nm})$ & $\sigma$ & GA & $\varepsilon$ & PDI \\
\hline pvdf $+1 \% \mathrm{Fe}_{3} \mathrm{O}_{4}$ & $-18.14-16.68$ & -17.62 & 0.0516 & 0.6435 & 0.0103 & 1.00 \\
\hline pvdf $+3 \% \mathrm{Fe}_{3} \mathrm{O}_{4}$ & $-17.89-16.73$ & -17.434 & 0.039 & 0.535 & -0.208 & -1.00 \\
\hline
\end{tabular}

Fig. 2 The size distribution function of $\mathrm{Fe}_{3} \mathrm{O}_{4}$ particles in the PVDF polymer matrix: a $\mathrm{PVDF}+1 \% \mathrm{Fe}_{3} \mathrm{O}_{4}$, b $\mathrm{PVDF}+3 \% \mathrm{Fe}_{3} \mathrm{O}_{4}$
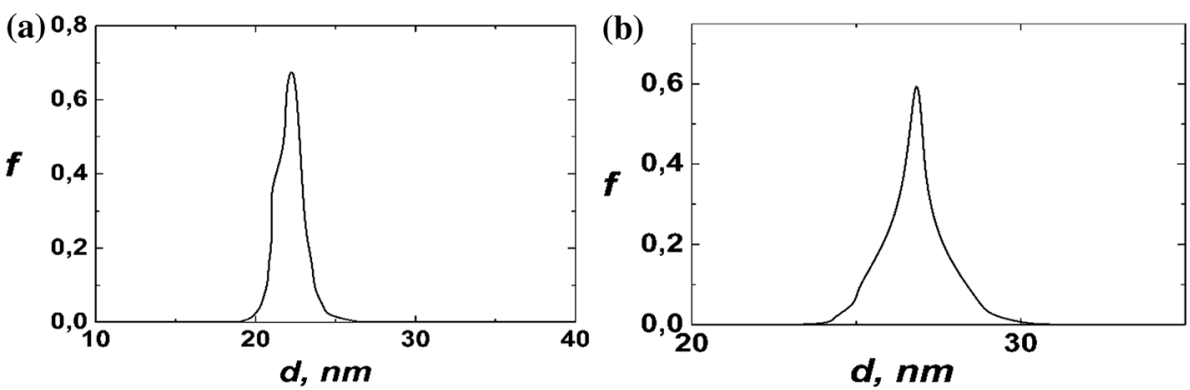

the concentration of the filler in the matrix increases, the viscosity of the medium changes and the coagulation condition changes as well.

The linear part of the $d(\varphi)$ dependence curve (low concentration) can be described by using the following equations:
$d_{\mathrm{Fe}_{3} \mathrm{O}_{4}}=6.67+5000.0 \cdot \varphi$

At high concentrations of the filler, the saturation occurs, reaching at the maximum value of the coagulated particle diameter, which can be described with an exponential law (Fig. 3a). 
Fig. 3 Dependence of the diameter of $\mathrm{Fe}_{3} \mathrm{O}_{4}$ nanoparticles on their concentration in polymer PVDF matrix. a High content of the filler; b low content of the filler
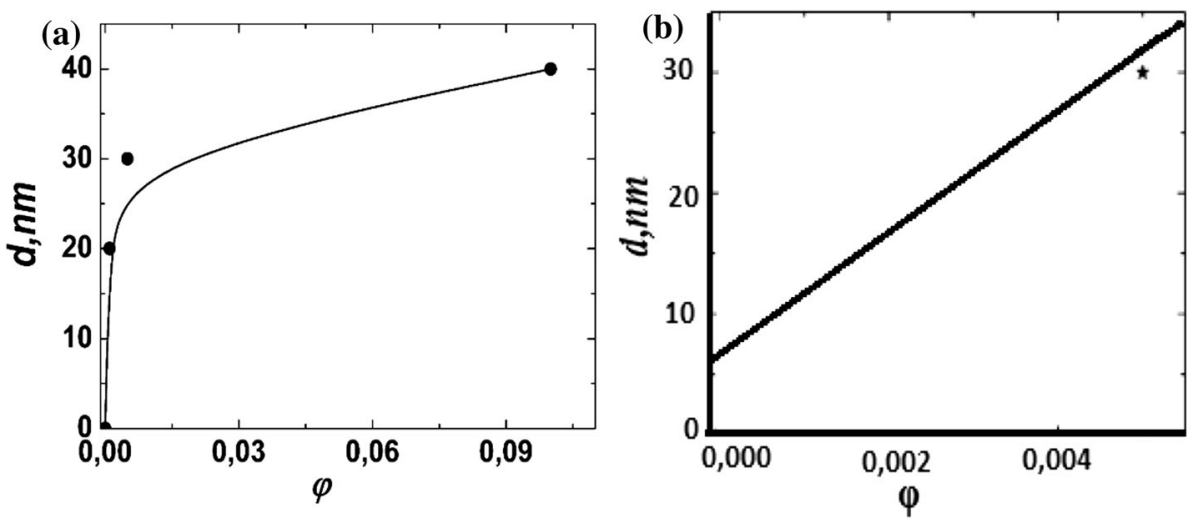

The theory of coagulation of nanoparticles in solutions at their low concentrations is known. According to this theory, the number of particles during coagulation decreases according to the law $[6,7]$

$\frac{\mathrm{d} n}{\mathrm{~d} t}=-k_{0} \cdot n^{2}$

The value of $\kappa_{0}$ is defined as:

$k_{0}=\frac{8 k T}{3 \eta}$

where $\eta$ is the viscosity of medium.

By the definition $\varphi_{\mathrm{s}}=\frac{V_{\mathrm{s}}}{V}$, where $V_{\mathrm{s}}, V$ is the volume of solid phase and medium, respectively. Since $V_{\mathrm{s}}=N \cdot V_{0}$ and $n=\frac{N}{V}=\frac{\varphi_{\mathrm{S}}}{V_{0 \mathrm{~s}}}$, we rewrite the last differential equation in the following form:

$\frac{\mathrm{d} V}{\mathrm{~d} t}=-k_{0} \varphi_{\mathrm{s}}$

The solution is $V_{0 t}=k_{0} \cdot \varphi_{\mathrm{s}}+V_{0}$. In the latter expression, if we substitute $d_{0 t}$, we will get the following formula:

$d_{0 t}=d_{0}\left(1+\frac{2 k_{0} \varphi_{\mathrm{s}} t}{\pi d_{0}^{3}}\right)$

The expression obtained is valid for low volume content of particle. As can be seen, the diameter of nanoparticles aggregated is a linear function of concentration, which is consistent with the previous results obtained (Fig. 3b). At high concentration of the filler, the last expression should be rewritten with the help of such a function that the value of the function should asymptotically approach to the fixed value " $d$ " when the value of the variable is high.

The values of " $k$ " were calculated with the help of (2. To determine the viscosity of the mixture, the Walter formula was used $[7,8]$ :

$\lg \lg \left(v_{\text {mixture }}+0.8\right)=\sum_{i=1}^{n} x_{i} \lg \lg \left(v_{i}+0.6\right)$

where $\gamma_{\text {mix }}$ and $\gamma_{i}$ are the kinematic viscosities of the mixture and the $\mathrm{i}$-th component, and $\mathrm{x}_{\mathrm{i}}$ is the fraction of the component in the medium. From the latter it is possible to determine the kinematic (further dynamic) viscosity of the mixture:

$v_{\text {mix }}=\exp \left(\exp \left(\sum_{i=1}^{n} x_{i} \lg \lg \left(v_{i}+0.6\right)\right)\right)$

The obtained results are consistent with AFM illustrations.

Morphology of the samples and the degree of dispersion of inorganic phase in the polymer matrix were investigated using atomic force microscopy (AFM) (Fig. 4). AFM observation showed that the size of the dispersed phases increases with increasing filler content in the nanocomposites. The sizes of $\mathrm{Fe}_{3} \mathrm{O}_{4}$ nanoparticles are 22, 33 and $45 \mathrm{~nm}$ for 1,3 and $6 \%$ volume content of filler, respectively. These results were found to be consistent with the results obtained from the theoretical calculations.

The magnetic properties of $\mathrm{PVDF}+\mathrm{Fe}_{3} \mathrm{O}_{4}$-based nanocomposites were studied by experimental and theoretical investigation. Figure 6 illustrates the experimental hysteresis loops of magnetic polymer composite materials with different volume contents of the filler.

$M(H)$ magnetization versus magnetic field curves of $\mathrm{PVDF}+\mathrm{Fe}_{3} \mathrm{O}_{4}$-based nanocomposites for different filler concentrations have a similar shape (superimposable). In this case, only the $0-50 \mathrm{KOe}$ section of the $M(H)$ curve is informative. The inset at the left panel demonstrates $M(H)$ magnetic curve of PVDF $+\mathrm{Fe}_{3} \mathrm{O}_{4}$-based nanocomposites at low magnetic field. However, the whole $m(H)$ curve of PVDF $+\mathrm{Fe}_{3} \mathrm{O}_{4}$ sample is coherent with a SPM behavior of this material. The inset on the left shows a small hysteresis loop close to $H=0$ which is within the margin of error (Table 3 ).

Figure $6 \mathrm{a}, \mathrm{b}$ shows the theoretical magnetization versus magnetic field curve and the magnetic susceptibility curve of a composite based on PVDF matrix and magnetite nanoparticles with size of $20 \mathrm{~nm}$. Numerical differentiation was carried out by the method $[9,10]$. 


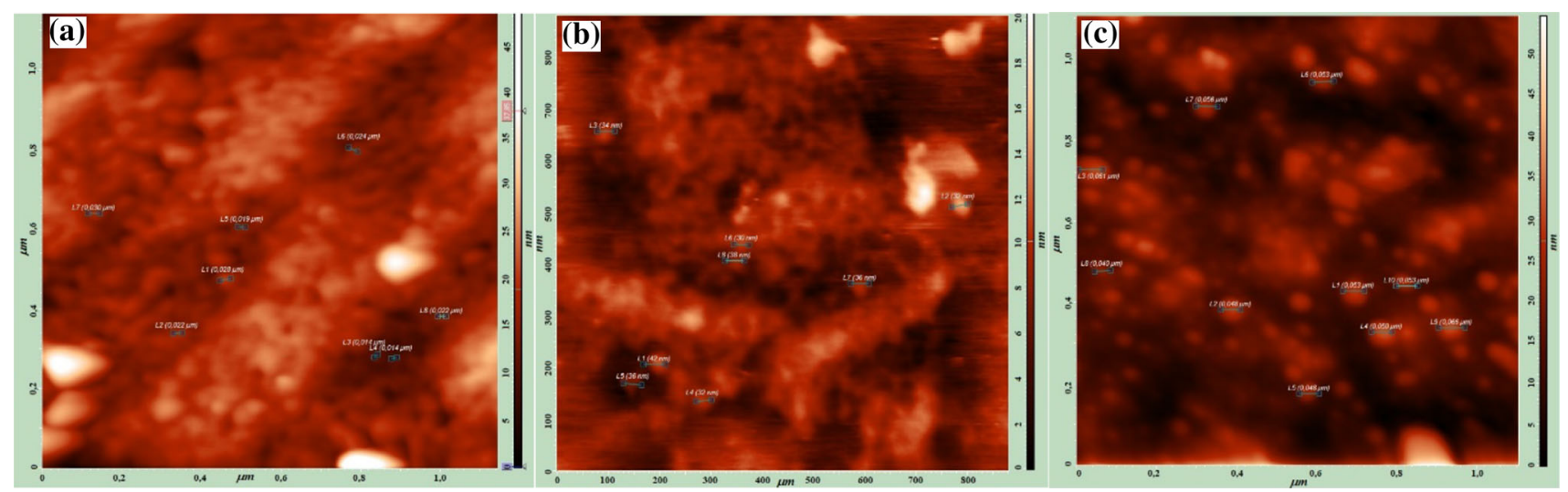

Fig. 4 AFM image of PVDF $+\mathrm{Fe}_{3} \mathrm{O}_{4}$ nanocomposites. a PVDF +1 vol\% $\mathrm{Fe}_{3} \mathrm{O}_{4}, \mathbf{b} \mathrm{PVDF}+3$ vol\% $\mathrm{Fe}_{3} \mathrm{O}_{4}$ and $\mathbf{c} \mathrm{PVDF}+6$ vol\% $\mathrm{Fe} \mathrm{O}_{4}$

Table 3 Results obtained from magnetic measurements

\begin{tabular}{lll}
\hline Sample & $\mathrm{Ms}$ & $\mathrm{Mr}$ \\
\hline $\mathrm{PVDF}+1 \mathrm{vol} \% \mathrm{Fe}_{3} \mathrm{O}_{4}$ & 2.24 & 0.022 \\
$\mathrm{PVDF}+3 \mathrm{vol} \% \mathrm{Fe}_{3} \mathrm{O}_{4}$ & 8.01 & 0.073 \\
$\mathrm{PVDF}+6 \mathrm{vol} \% \mathrm{Fe}_{3} \mathrm{O}_{4}$ & 8.12 & 0.19 \\
\hline
\end{tabular}

As can be seen from Fig. 6a, b, the magnetization curves have the hysteresis loop. This is typical for particles which have many magnetic domains. The hysteresis parameters of the nanocomposites (saturation magnetization, residual magnetization, coercive force, etc.) depend on the size and concentration of $\mathrm{Fe}_{3} \mathrm{O}_{4}$ particles. As the size and concentration of $\mathrm{Fe}_{3} \mathrm{O}_{4}$ particles increase, the number of magnetic domains increases in the both particle and medium. As can

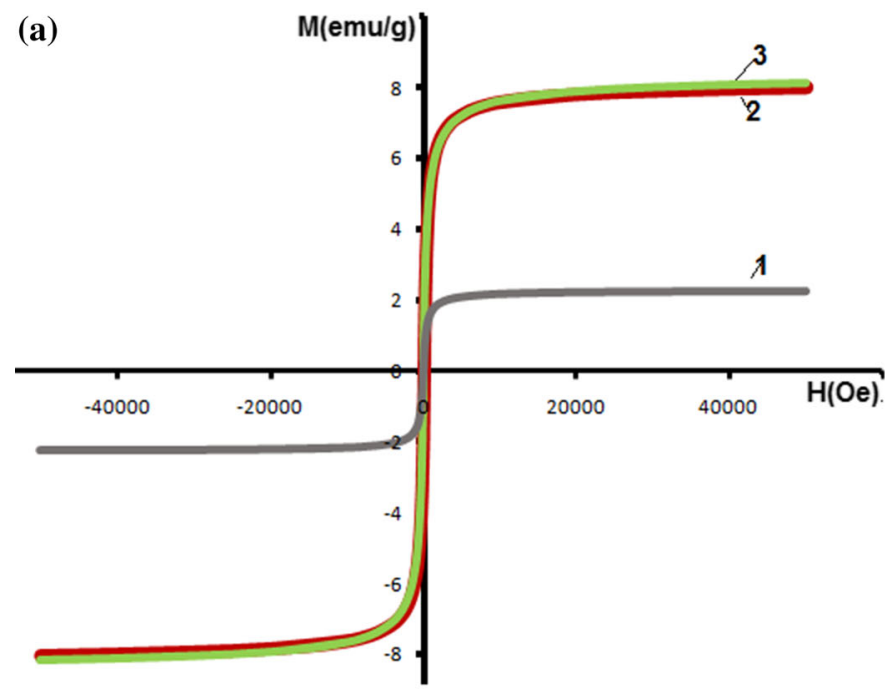

Fig. $5 M(H)$ Magnetization versus magnetic field curves of $\mathrm{PVDF}+\mathrm{Fe}_{3} \mathrm{O}_{4}$-based nanocomposites. a (1) $\mathrm{PVDF}+1 \mathrm{vol} \%$

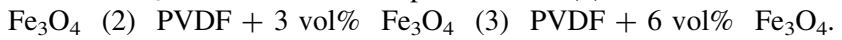

be seen from Figs. 5 and $6 a$, the magnetic field dependence of the magnetization obtained from the experiments and calculated theoretically is close to each other for low magnetic field.

It is known that for dispersed systems, during the magnetization, the value of the saturation magnetization can be given by the following equation:

$M_{\mathrm{s}}=\varphi_{m} M_{\mathrm{s}}^{\prime}$

where $M_{\mathrm{s}}, M_{\mathrm{s}}^{\prime}$ are the saturation magnetizations of the composite and the filler, respectively, and $\varphi_{\mathrm{m}}$ is the volume content of the filler.

To explain this, suppose that the geometric sizes of the nanoparticles $(\mathrm{d} t)$ are not equal to the magnetic sizes of the nanoparticles $(\mathrm{d} m)$.

For comparison of magnetic sizes with the geometric sizes of nanoparticles, we use the following equations:

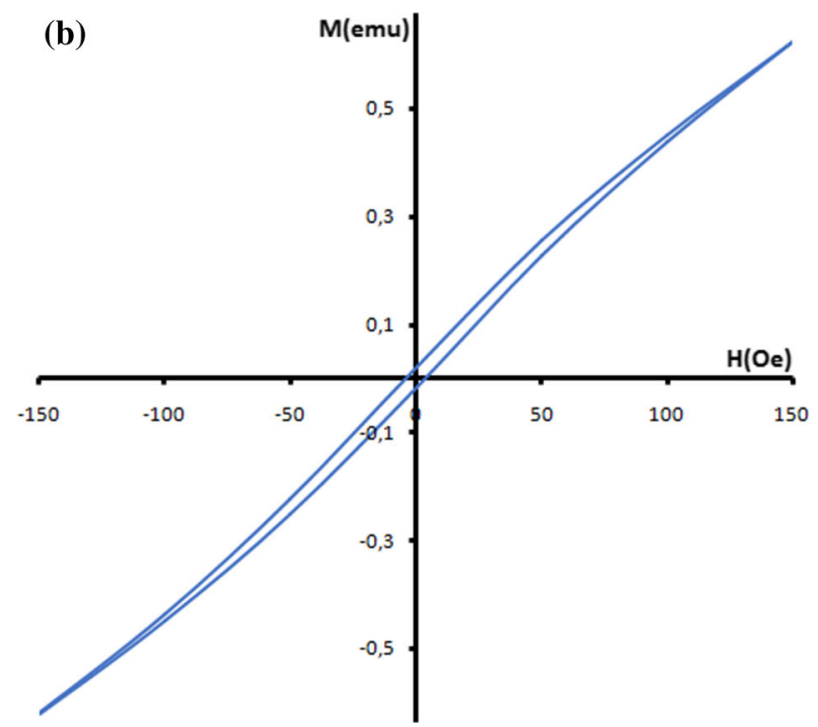

b Experimental magnetization versus magnetic field $M(H)$ curves of PVDF $+\mathrm{Fe}_{3} \mathrm{O}_{4}$-based nanocomposites under low magnetic filed for $\mathrm{PVDF}+1 \% \mathrm{Fe}_{3} \mathrm{O}_{4}$ 
Fig. 6 Theoretically calculated magnetic properties of $\mathrm{PVDF}+\mathrm{Fe}_{3} \mathrm{O}_{4}$ nanocomposites. a Magnezitation versus magnetic field $M(H)$ b susceptibility versus magnetic field curves $\chi(H)$

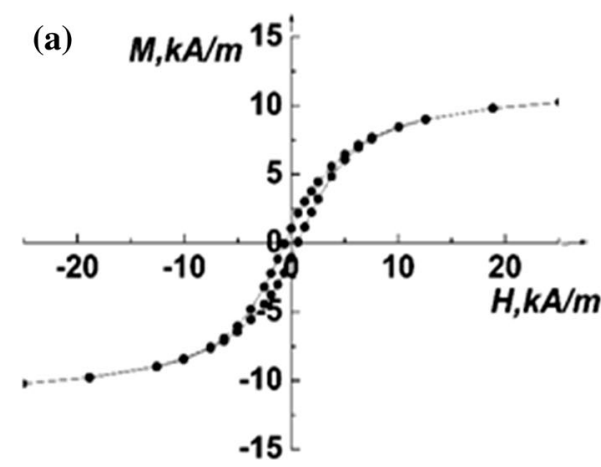

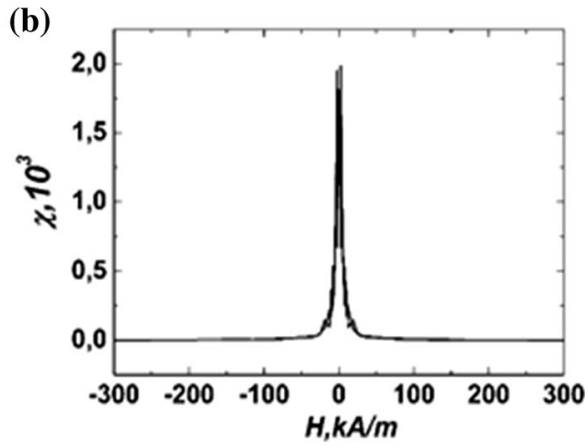

$V_{t}=N_{t} \cdot \frac{\pi}{6} \overline{d_{t}^{3}}$

where $\varphi_{t}, \varphi_{\mathrm{m}}$ and $V_{\mathrm{m}}$ can be given as below:

$$
\begin{aligned}
\varphi_{t} & =V_{t} / V, \varphi_{\mathrm{m}}=V_{\mathrm{m}} / V \text { and } V_{\mathrm{m}}=N_{\mathrm{m}} \cdot \frac{\pi}{6} \overline{d_{\mathrm{m}}^{3}} \\
& =N_{\mathrm{m}} \cdot \frac{\pi}{6} \overline{\left(d_{t}-x\right)^{3}}
\end{aligned}
$$

where $V, V_{t}$ and $V_{\mathrm{m}}$ are the volumes of medium, solid and magnetic phase, respectively. Then, by using the expression for the concentration of the magnetic phase, we obtain the relation between geometrical and magnetic phase:

$\varphi_{t} / \varphi_{\mathrm{m}}=\overline{d_{t}^{3}} / \overline{\left(d_{t}+x\right)^{3}}=\alpha$

After the manipulation of the last expression, we get the cubic equation:

$\overline{x^{3}}-3 \cdot \overline{d_{g} x^{2}}+3 \cdot \overline{d_{g}^{2} x}-(1-\alpha) \overline{d_{g}^{3}}=0$

The last equation was solved by the Cardan method [11]. Table 4 reports the results obtained from cubic equation. It should be noted that the magnetic properties of nanocomposites are independent on the type of the polymer.

It can be concluded that the thickness of the polymer layer around nanoparticle rises with the increasing size of the nanoparticles and it also depends on the properties of the medium (molecular weight, polarity, viscosity, etc.).

It can also be seen from the table that in all cases the particle size increases, except for $d=20 \mathrm{~nm}, \varphi=0.03$,

Table 4 The result obtained from cubic Eq. (4)

\begin{tabular}{lllc}
\hline$d(\mathrm{~nm})$ & $\varphi_{\mathrm{t}}$ & $\varphi_{\mathrm{m}}$ & $x(\mathrm{~nm})$ \\
\hline 20 & 0.01 & 0.371 & 46.7 \\
40 & 0.01 & 0.323 & 87.3 \\
20 & 0.03 & 0.0254 & -1.07 \\
30 & 0.03 & 1.33 & 76.9 \\
30 & 0.06 & 1.35 & 55.4 \\
40 & 0.07 & 1.35 & \\
\hline
\end{tabular}

where the size decreases (i.e., the oxidization of the surface layer is close to the lattice parameter of the particle, $a=0.839 \mathrm{~nm})$.

\section{Conclusion}

It was found that when the $\mathrm{Fe}_{3} \mathrm{O}_{4}$ particles are introduced into the polymer matrix, their coagulation takes place. The increase in the particle size depends on their concentration in the polymer matrix, the type of polymer (polar, nonpolar, viscosity, etc.), reaction temperatures, etc. When $\mathrm{Fe}_{3} \mathrm{O}_{4}$ particles are introduced into the polymer matrix, at the same time with the coagulation, the oxidation of their surface layer also occurs and the magnetic size decreases and hence the magnetic characteristics are reduced. It becomes clear that the magnetic hysteresis parameters depend on the size and concentration of $\mathrm{Fe}_{3} \mathrm{O}_{4}$ particles in the polymer matrix. Theoretical calculations were compared with experimental results obtained from $M(H)$ measurements. At the same time, it was shown that the magnetic field dependence of the magnetization obtained from the experiments and calculated theoretically is close to each other for low magnetic field. With increasing nanoparticle size, the difference between theoretical and experimental values increases. It is related to the fact that unlike to the reality, theoretically the magnetite nanoparticles are accepted to be single-domained.

Open Access This article is distributed under the terms of the Creative Commons Attribution 4.0 International License (http://creative commons.org/licenses/by/4.0/), which permits unrestricted use, distribution, and reproduction in any medium, provided you give appropriate credit to the original author(s) and the source, provide a link to the Creative Commons license, and indicate if changes were made.

\section{References}

1. Ramazanov, M.A., Hajiyeva, F.V., Maharramov, A.M., Di Palma, L., Sannino, D., Makoto Takafuji, H.M., Mammadov, U., Hasanova, A., Shirinova, H.A., Bayramova, Z.A.: New magnetic 
polymer nanocomposites on the basis of isotactic polypropylene and magnetite nanoparticles for adsorption of ultra high frequency electromagnetic waves. Polym. Plast. Technol. Eng. (2017). https://doi.org/10.1080/03602559.2017.1320721

2. Shirinovaa, H., Di Palma, L., Sarasinib, F., Tirillòb, J., Ramazanov, M.A., Hajiyevaa, F., Sanninoc, D., Polichettid, M., Galluzzid, A.: Synthesis and characterization of magnetic nanocomposites for environmental remediation. Chem. Eng. Trans. 47, 103-108 (2016)

3. Stabik, J., Chrobak, A., Haneczok, G., Dybowska, A.: Magnetic properties of polymer matrix composites filled with ferrite powders. Arch. Mater. Sci. Eng. 48(2), 97-102 (2011)

4. Ali-zade, R.A., Ramazanov, M.A., Sadykhov, R.Z.: Size distribution of magnetite nanoparticles in a polymer matrix. Ukr. J. Funct. Mater. 16(2), 183-189 (2009)

5. Shchukin, E.D., Pertsov, A.V., Amelina, E.A., Zelenev, A.S.: Colloid and Surface Chemistry, vol. 12, p. 774. Elsevier Science, Amsterdam (2001)

6. Gerasimov, Y.I., Dreving, V.P., Eremin, E.N., Kiselev, A.V., Lebedev, V.P., Panchenkov, G.M., Shlygin, A.I.: Kursfizicheskojhimii [Course in Physical Chemistry], vol. 2, p. 624. Khimiya, Moscow (1973). (In Russian)
7. Evdokimov, I.N., Losev, A.P.: Absence of additivity of properties of petroleum mixtures. Drill. oil Technol. 1, 32-33 (2012)

8. Ramazanov, M.A., Alizade, R.A., Maharramov, A.M., Hajiyeva, F.V., Sultanova, J.R., Shirinova, H.A.: Theoretical and experimental study of the magnetic properties and size of distribution of PVDF + Fe based nanocomposites. J. Inorg. Organomet. Polym. Mater. (2018). https://doi.org/10.1007/s10904-018-0863-2

9. Bakhvalov, N.S., Zhidkov, N.P., Kobelkov, G.M.: Numerical Methods. Nauka, Moscow (1975)

10. Krylov, V.I., Bobkov, V.V., Monostyrny, P.I.: Computational Methods, vol. 2, p. 399. Nauka, Moscow (1977). (Russian)

11. Mathematical Handbook for Scientists and Engineers: Definitions, Theorems, and Formulas for Reference and Review (Dover Civil and Mechanical Engineering) [Granino A. Korn, Theresa M. Korn], p. 832. Nauka, Moscow (1973)

\section{Publisher's Note}

Springer Nature remains neutral with regard to jurisdictional claims in published maps and institutional affiliations. 